\title{
Efficient Dynamic Economic Load Dispatch Using Parallel Process of Enhanced Optimization Approach
}

\author{
S. Hemavathi*, N. Devarajan \\ Department of EEE, Government College of Technology, Coimbatore, India \\ Email: "hemaphd@gmail.com
}

Received 20 February 2016; accepted 15 March 2016; published 25 August 2016

Copyright (C) 2016 by authors and Scientific Research Publishing Inc.

This work is licensed under the Creative Commons Attribution International License (CC BY). http://creativecommons.org/licenses/by/4.0/

c) (i) Open Access

\begin{abstract}
In Dynamic Economic Load Dispatch (DELD), optimization and evolution computation become a major part with the strategy for solving the issues. From various algorithms Differential Evolution (DE) and Particle Swarm Optimization (PSO) algorithms are used to encode in a vector form and in sharing information and both approaches are based on the master-apprentice mechanism for the Dual Evolution Strategy. In order to overcome the challenges like the clustering of PSO, optimization problems and maximum and minimum searching, a new approach is developed with the improvement of searching and efficient process. In this paper, an Enhanced Hybrid Differential Evolution and Particle Swarm Optimization (EHDE-PSO) is proposed with Dynamic Sigmoid Weight using parallel procedures. A hybrid form of the proposed approach combines the optimizing algorithm of Enhanced PSO with the Differential Evolution (DE) for the improvement of computation using parallel process. The implementation and the parallel process are analyzed and discussed to gather relevant data to show the performance enhancement which is better than the existing algorithm.
\end{abstract}

\section{Keywords}

Differential Evolution, PSO, Hybrid, Load Dispatch, Sigmoid Weight, Optimal Solution

\section{Introduction}

Nowadays, the main scope of optimization is determining the best solution for a problem under constraints. Several researchers over the years conclude with different solutions to linear and non-linear optimization problems. The mathematically fitness function is involved in the optimization problem in defining under a set of

${ }^{*}$ Corresponding author.

How to cite this paper: Hemavathi, S. and Devarajan, N. (2016) Efficient Dynamic Economic Load Dispatch Using Parallel Process of Enhanced Optimization Approach. Circuits and Systems, 7, 3260-3270.

http://dx.doi.org/10.4236/cs.2016.710278 
constraints to represent the solution space for the issues.

Unfortunately, the traditional optimization techniques evaluate the first derivatives to detect the optima on a known constrained surface. Because of the difficulties in evaluating the first derivatives, free optimization algorithms have emerged to overcome the difficulty. The optimization problems are characterized as an intellectual search issue, where the agents are engaged to regulate the optima on a search landscape. It represents the constrained surface for the optimization issue.

In the later quarter of the twentieth century, an evolutionary search algorithm is come up with a solution to the non-linear optimization problems. By the inspiration of natural adaptations of the biological species, the Darwinian Theory is proposed through Genetic Algorithms (GA). It disseminates the GA theory and validates the biological crossovers and chromosome mutations, which is used to improve the solution's quality over successive iterations.

In mid 1990s, Particle Swarm Optimization (PSO) is an alternative solution for the complex non-linear optimization problem which is replicating the mutual behaviour of bird flocks. At the same time, a suitable differential operator named as Differential Evolution (DE) is defined to replace the alternative operators in the place of mutation operators and classical crossover of GA. In DE and PSO gradient information of the function is not required and it uses only primitive mathematical operators and is conceptually easy.

Implementation of computer language is very easy and it requires only minimal parameter tuning. There is no decline in the algorithm performance with the growth of the search space dimensions. There is a great role in the algorithm's popularity within the machine intelligence and cybernetics domain.

For a lot of problems, a simple evolutionary algorithm is good enough to find the covet solution. There are several types of problems in the direct evolutionary algorithm, because sometimes it may fail to obtain a convenient (optimal) solution. This is clearly craved the necessary of hybridization of evolutionary algorithms with the other optimization algorithms, heuristics, machine learning techniques, etc. Some of the imaginable reasons for hybridization are the convergence speed of the evolutionary algorithm, solution quality, which is integrated as part of a larger system.

Evolutionary Computation (EC) inhales the ideas, which are activated from the community-based animal's behavior such as birds, ants and bees. The traditional algorithm uses the evolution computation technique and is applied to scheduling, route planning, network optimization, data mining and decision support. The foraging behavior of birds motivates the PSO because of simple procedure implementation, fast convergence and less parameters setting.

Therefore, PSO has been standard in various fields. Although PSO is capable of retaining some demanding problems like premature merging and easily drop into regional optimum, the DE approach is used for the random search, less setting of parameters, better performance and appropriate to high-dimensional difficult optimization issues. Also, DE has various faults with unbalanced convergence in the prior period and easy droplet in the regional optimal.

In recent days, many noticeable optimization theories are in practical. There are very few people who are experts in algorithms and some of them are also proficient in parallel programming. The DE techniques are having many local optima, which are partially surrounded by a very flat surface and can handle the issues. This makes the solution go out of range and quantifying the quality of the potential solutions will become difficult in the function. In addition, PSO have difficulties with easy local minimum occurring, search stage slow convergence or weak local search ability. These are the stable reasons to represent in DE and PSO.

In this study, based on the approaches the dual evolution algorithm is raised with relayed sharing of information and the benefits of the two algorithms are carried out in the proposed approach. The rest of the paper is organized with following sections. In Section 2, the literature survey of the optimization approach is discussed and the proposed work is explained in Section 3. The simulation results and the conclusion are presented in Section 4 and Section 5 respectively.

\section{Related Work}

In this section, the literature survey of the various optimization approaches used for efficient Dynamic Economic Load Dispatch (DELD) is discussed. Practically, the power plants are located far away from the centre of loads and the fuel cost for the transportation is different. Economic Load Dispatch (ELD) is the generator scheduling used to minimize the generator's operating cost which is subjected to the equality constraint of power 
balance within the minimum and maximum operating limits of the generating units [1].

An application of BAT is presented for generalizing multi-objective optimization problem in power system. Emissions produced from fossil-fuelled power plant are considered to be a hazardous impact on the environment. The economic dispatch minimizes the total fuel cost can no longer considered to be a single objective. The BAT algorithm application is based on mathematical modelling to solve economic, emission and combined economic emission dispatch problems by a single equivalent objective function [2].

The soft computation method has achieved supplementary concentration in successful and sensible applications. By using Particle Swarm Optimization (PSO) Algorithm, an attempt is made to find the minimum cost. The data with the Loss coefficients with the max min power limit and cost function. PSO and Simulated Annealing (SA) are applied to find out the minimum cost for different power demand. By comparing the obtained results with the previous technique, it is evident that the results provide better convergence characteristic [3].

Reactive power dispatch (RPD) is considered to be one of the important tasks in the operation and control of the power system. To solve RPD problem, an efficient and reliable evolutionary-based approach is implemented and for optimal settings of RPD control variables, differential evolution (DE) approach is used [4]. By implementing Hybrid Bacterial Foraging-Differential Evolution optimization algorithm, economic load dispatch (ELD) problem is solved. In the hybrid approach, computational chemotaxis of BFOA is viewed as astochastic gradient search and coupled with DE type mutation and crossover of the optimization agents. Non-convex economic load dispatch problems along with different constraints like transmission losses, dynamic operation constraints (ramp rate limits) and prohibited operating zones can be easily solved using this method [5].

The Economic dispatch problem (ED) is solved by an approach, which is based on a hybrid algorithm consisting of Genetic Algorithm (GA), Pattern Search (PS) and Sequential Quadratic Programming (SQP) techniques. GA is the main optimizer of the algorithm and PS, SQP is used to fine tune the results of the GA so that it increases the efficiency of the solution [6] [7]. To solve the economic load dispatch (ELD) problem in power systems, an improved coordinated aggregation-based particle swarm optimization (ICA-PSO) algorithm is introduced in this paper. Whereas in the ICA-PSO algorithm, all the particles have a memory in its best position and other particles can attract them with best achievement, when they move randomly. So, the population size is increased and search intervals for the particles is adaptively selected [8] [9].

An important task in the power system is Economic Dispatch. The main process of this task is to allocate the committed unit's generation. So that energy requirements are reduced and the constraints are imposed. In power generation, Maclaurin series based Lagrangian method (MSL) is applied with valve-point effect to resolve the DED issues by the rate limits consideration [10]. The hybrid approaches of Particle Swarm Optimization (PSO) with Genetic Algorithm (GA) are implemented to solve optimization problem. The PSO and GA are population based heuristic search technique. In standard PSO, the non-oscillatory route can quickly cause a particle to stagnate [11].

The genuine stochastic optimisation approach determines the feasible optimal solution of the economic dispatch (ED) problem. The proposed optimisation algorithm self-tuning hybrid differential evolution (self-tuning HDE) used to increase the global optimum search and utilizes the concept of the 1/5 success rule of evolution strategies (ESs) in the original HDE [12].

The Evolutionary algorithm based method is implemented for solving the economic load dispatch (ELD) problem. The main scope is to minimize the nonlinear function for the total fuel cost of thermal generating units [13]. Differential evolution, which is a new population based technique for obtaining an increasing attraction in many engineering applications, especially for power engineering. The conventional evolutionary algorithm depends on the predefined probability distribution function for mutation. To provide efficient global optimization capability, object vector's differences will be passed to the objective functions topographical information [14].

Economic Dispatch (ED) is considered to be one of the important functions of the power system operation and control. It determines the optimal real power settings of generating units. Practically, the generator's fuel cost can be represented as a quadratic function of real power generation. Because of valve point loading, there is a discontinuity in thermal power plants' fuel cost curves. Particle Swarm Optimization (PSO) is implemented for solving the ED problem of generating units having non-smooth fuel cost functions with ramp rate limits. The comparison of the obtained result with the previous work dedicatedly proves the method to be feasible and efficient [15].

A novel multi objective evolutionary algorithm for environmental/economic power dispatch (EED) optimization problem is discussed. With both equality and inequality constraints, EED problem is formulated as a nonli- 
near constrained multi objective optimization problem. To handle the problem, a new non dominated sorting genetic algorithm based approach is proposed. It employs the diversity-preserving mechanism to reduce the premature convergence. Also, employ in searching the bias problems and to produce a well distributed Paretooptimal set of non-dominated solutions. To provide the decision maker, a hierarchical clustering algorithm is imposed by representative and manageable Pareto-optimal set. To obtain the best solution over the trade-off curve, fuzzy set theory is applied [16].

\section{Proposed Work}

In this section, the explanation of the proposed approach EHDE-PSO is considered with the modified Sigmoid Weight. The technique proposed in the paper is based on EPSO and mutation operators of the HDE for global optimal solution and also to reduce the computational time. For optimization improvement in solving NP-Hard problems, a hybrid algorithm is proposed as Enhanced Hybrid Differential Evolution and Enhanced Particle Swarm Optimization with Dynamic Sigmoid Weights. However, it is a combine process of Hybrid DE (HDE) and Enhanced PSO (EPSO) algorithms in parallel. By parallel process the performance of speed and optimization is improved.

The hybrid version of the algorithm is modified for efficient access in Dynamic Economic Load Dispatch. The EPSO is performed with the weight when the iteration is executing else DE is performed and it will continue till the certain condition satisfied. The HDE is involved in the process of selection, mutation and recombination and passed to EPSO for the early convergence deficiency improvement. By easily trapping the local EPSO optimum, the slow convergence in HDE can be resolved and improved. To enhance the searching performance, the concept of dynamic sigmoid weight is applied.

The evolution of the proposed approach is done with the strategy of iteration and shares the outcomes by interchanging iteration to another. The speed of EPSO is improved by the involvement of modified dynamic sigmoid function. The general function of sigmoid increasing $\left(W_{i}\right)$ and decreasing $\left(W_{d}\right)$ is considered by adding modify sharpness and it is estimated as follows.

$$
\begin{aligned}
& W_{i}=\frac{(W b-W f)}{1+\mathrm{e}^{-(\log (g e n)-2)(i-n * g e n)}}+W f \\
& W_{d}=\frac{(W b-W f)}{1+\mathrm{e}^{(\log (g e n)-2)(d-n * g e n)}}+W f
\end{aligned}
$$

Where, the beginning weight function is represented as $W b$ and the finishing stage as $W f . W_{i}$ and $W_{d}$ indicates the weight function for the inertia interchange and accelerator weights. Here, " $i$ " and " $d$ " are the iteration process, gen as maximum population, $n$ as constant sigmoid $(0.75,0.5$ or 0.25$)$.

According to the function it constructs S-shape and for all iteration the accelerator and the weight. In the beginning, if the value is highest and decreased as per the progress of the algorithm then it is decreasing function. If begin will small values and increase the value to higher during the execution of an algorithm is defined as increasing function. The modified sigmoid function is shown in Figure 1.

As per the proposed approach, the objective utility of equality and inequality issues has underestimated the operating cost of the generating units.

The objective is:

$$
\operatorname{MinC}=\sum_{i=1}^{o g}\left(a_{n} P_{g n}^{2}+b_{n} P_{g n}+c\right) \text { Rs } / \text { MWh }
$$

The constraint balance of power is enhancing the inequality and equality and it is mentioned as generation restrictions. The value point cost effects are managed by using the sinusoidal function and signified as

$$
\begin{gathered}
C_{i}\left(D_{i}^{t}\right)=1 /\left(2 * a_{i}+b_{i} D_{i}^{t}+c_{i}\left(D_{i}^{t}\right)^{2}+\left|d_{i} \sin \left(e_{i}\left(D_{i}^{\min }-D_{i}^{t}\right)\right)\right|\right) \\
\sum_{i=1}^{N} P B^{t}=D^{i}+\operatorname{Loss}^{i} \\
\operatorname{Loss}^{i}=\sum_{j=1}^{o g} \sum_{i=1}^{o g} P_{r j} L_{j i} P_{r i}
\end{gathered}
$$

The Real power generating units is limited by the bounds of lower and upper with ramp rate limits according 


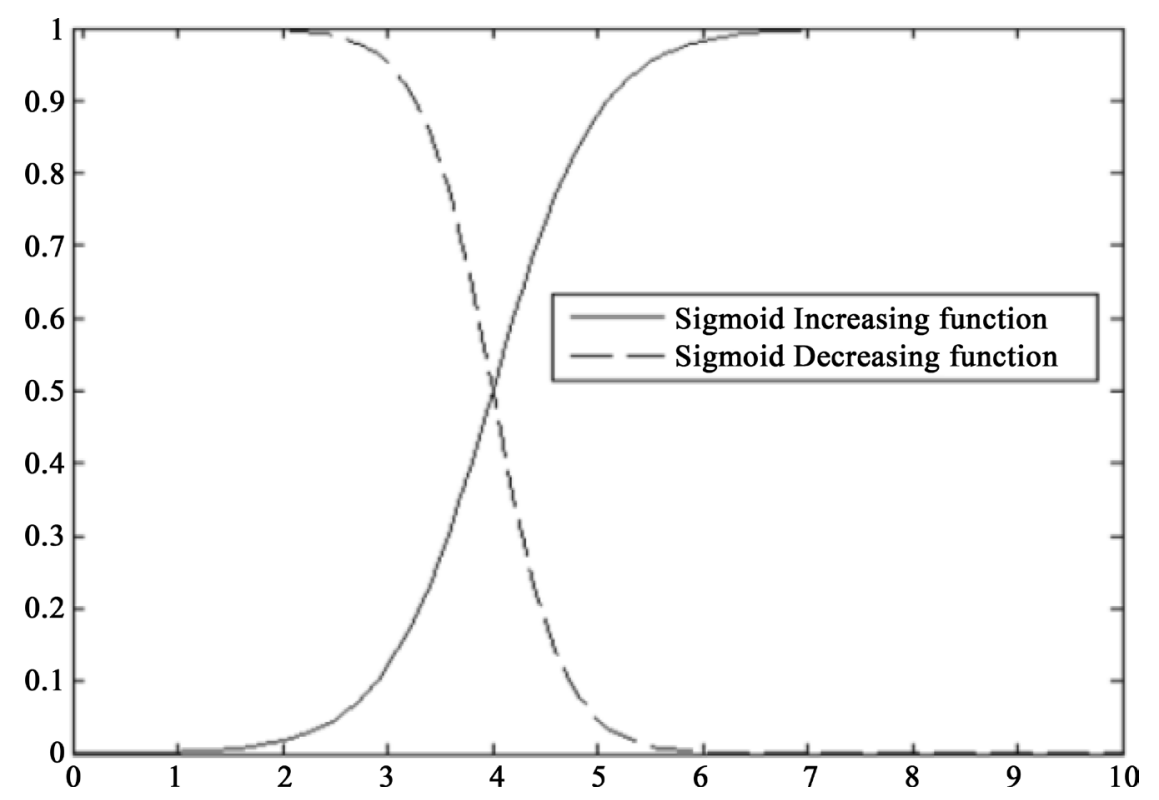

Figure 1. Sigmoid inertia weight (increasing and decreasing).

to the interval of time during generating units is as follows

$$
\begin{aligned}
& P_{g n}^{\min } \leq P_{g n} \leq P_{g n}^{\max } \\
& P_{g n}-P_{g(n-1)} \leq U_{n} \\
& P_{g(n-1)}-P_{g n} \leq L_{n}
\end{aligned}
$$

For finding generating unit best value the following equation is processed to obtain the outcomes and update the records of the unit position and velocity.

$$
\begin{aligned}
& V_{i j}^{(t+1)}=C F *\left(\omega * v_{i j}+c 1 * \operatorname{rand}\left(P_{i j}^{b e s t}-P_{i j}\right)+C 2 * \operatorname{rand}\left(G_{g i}^{b e s t}-P_{i j}\right)\right) \\
& C F=\frac{2}{2-c-\sqrt{c^{2}-4 * c}} \\
& \omega=\omega_{\text {max }}-\frac{\omega_{\text {max }}-\omega_{\text {min }}}{\text { iter }_{\text {max }}} * t
\end{aligned}
$$

The proposed algorithm combines HDE into EPSO by separating the logic algorithm. The separation procedure takes place by splitting the different round of work by odd and even round. For better solution particle is shared and most of the time the PSO value becomes the local value. The proposed algorithm is processed step by step by passing a particle to the EPSO, which is used to separate the particle into many groups. After grouping, it computes one round and the obtained result is passed to HDE to perform with the Mutation and Crossover, and the new outcome is forwarded to EPSO until it reaches the maximum generation. The mutation operators enhance the exploration of diversity performance of the EPSO by implementing the mutation operator scenario.

$$
V_{i j}^{(t+1)}=S F *\left(\left(P_{k}^{(t-\beta)}-P_{i j}^{(t)}\right)-\left(P_{q}^{(t-\beta)}-P_{i j}^{(t)}\right)-\left(P_{r}^{(t-\beta)}-P_{i j}^{(t)}\right)\right)
$$

where, $k, q$ and $r$ are the random process of particle index from the set of population and $k \neq q \neq r ; \beta$ represent the iteration of the previous and the scaling factor is indicated as SF, which is a real value between 0.1 and 2 . The various populations' amplification is controlled by SF for outflow of local solutions.

The procedure of the proposed EHDE-PSO is accessible as follow and the particle is defining the solution of encoded in the form of vectors. According to the flow the optimal solution is obtained. Figure 2 shows the flow work of the proposed approach. 


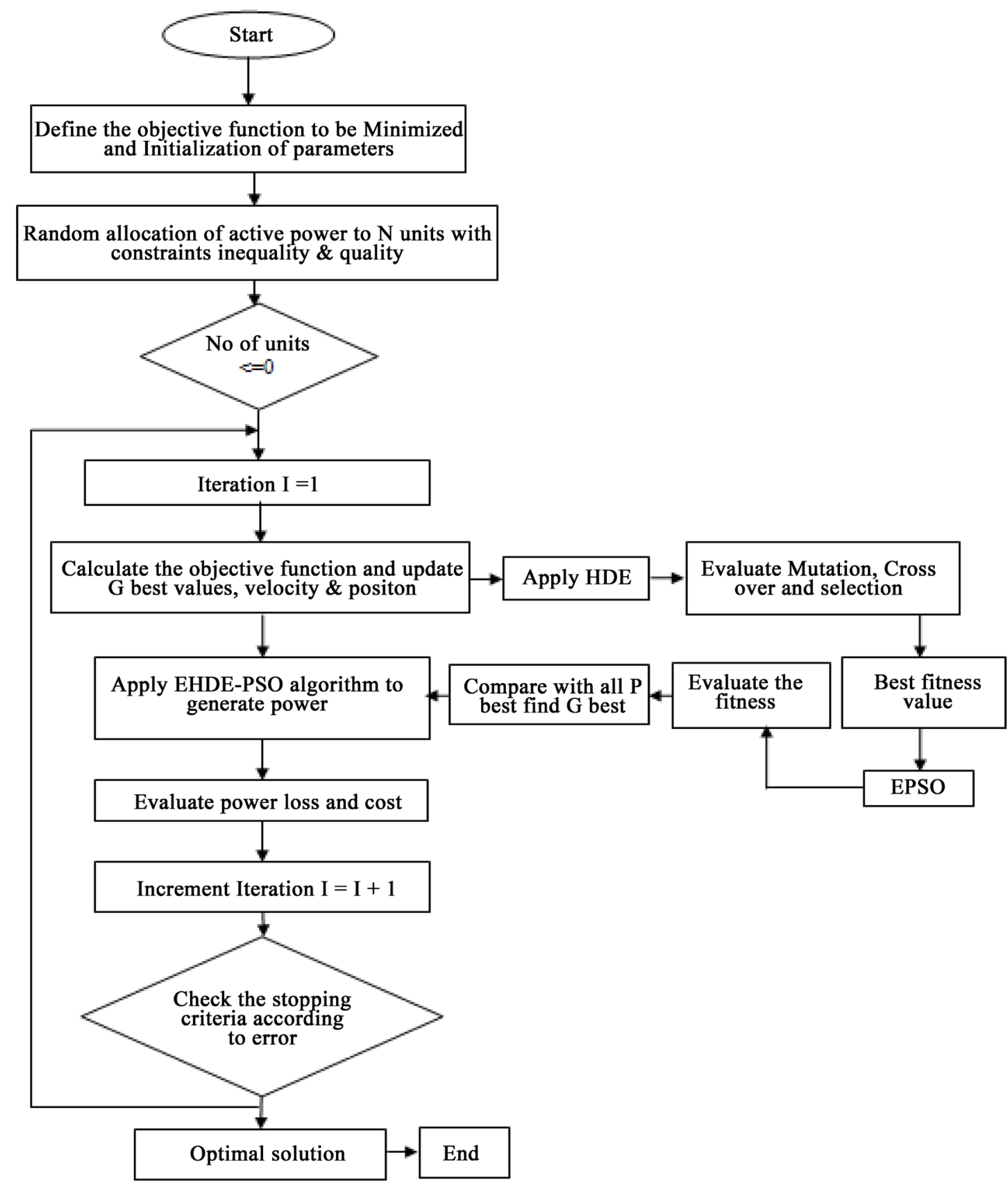

Figure 2. Flow work of proposed approach.

1. Random solution Initialization is taking place by placing particles in the space of solution $P(t)$ and the parameters are initialized. Here, $t$ is represents the iteration function.

2. If the iteration is zero or even, for optimization the proposed EPSO is applied with the modified sigmoid weight. Then it passed to a next generation by increasing the iteration by 1 .

3. If it is odd generating, then the proposed HDE functions will be performing.

4. In the population of DE, the Particles of EPSO are used. For the reverse of a parent generation chromosome is called by each solution.

5. In the HDE replication process, two chromosomes are randomly chosen for a trial vector. Normally eight trial vector is needed.

6. The fitness is then evaluated and the chromosome selects the highest value of fitness for next generation. 
7. Until the population is as same as original, the reproduction process will be repeated from step 2-6.

8. The solution of fitness is evaluated and checks the criteria of termination.

9. If it reaches the criterion, then the process will be stopped and update the new population of EPSO local best and global best.

10. Repeat steps 2-9.

As per procedure, first initialization is taking place and then the operation of both algorithms is separated. The fitness value is compared to choose the better one to start up the evolutionary process. As per separation, the algorithm is competing to process into sharing mechanism in even generating and it determines the master in the next generation. In odd generating, the particle is shared and sorted for the performances improvement and convergence, also act as master- apprentice. Finally the outcome of the optimal solution is obtained only if it reaches the terminated condition else continue with the iteration stage.

\section{Simulation Results}

In this section, the simulation results of the EHDE-PSO approach is carried out with the analysis of performances with the existing. The proposed algorithm is simulated and obtained the results using MATLAB. The measurement of the quality needs the reversal of gradient instead of difficult tasks. It reduces the complexity of the system with the objective function.

Moreover, the system is simulated for 6 units as given below. The cost and the power loss function are illustrated for the six unit test system and show the effectiveness of the approach then the existing. As shown in Table 1, in the generated power system the input is for each unit with the limitation of ramp and load for each scheduling period of time interval demand.

The proposed algorithm is examines and tested using the 6-unit system generation. Table 2 shows the performances of power generating for 6 units. Table 3 shows the better performance analysis in the cost and power loss of the proposed system than the existing. The fuel cost convergence for 6 units load demand (500, 700, 1000, 1200, 1350 and 1450) is simulated and plotted as shown in Figures 3-8 respectively.

Table 1. Characteristics of generation units.

\begin{tabular}{|c|c|c|c|c|c|c|c|}
\hline \multirow{2}{*}{ Unit i } & $a_{i}$ & $b_{i}$ & $c_{i}$ & $e_{i}$ & $f_{i}$ & $\mathbf{P}_{\mathbf{i}}^{\min }$ & $\mathbf{P}_{i}^{\max }$ \\
\hline & (\$/MW'h) & (\$/MWh) & $\$ / h$ & $\$ / h$ & $\mathrm{rad} / \mathrm{MW}$ & MW & MW \\
\hline 1 & 240 & 7 & 0.007 & 450 & 0.041 & 100 & 500 \\
\hline 2 & 200 & 10 & 0.0095 & 600 & 0.036 & 50 & 200 \\
\hline 3 & 300 & 8.5 & 0.0090 & 320 & 0.028 & 80 & 300 \\
\hline 4 & 150 & 11 & 0.0090 & 260 & 0.052 & 50 & 150 \\
\hline 5 & 200 & 10.5 & 0.0080 & 280 & 0.063 & 50 & 200 \\
\hline 6 & 120 & 12 & 0.0075 & 310 & 0.048 & 50 & 120 \\
\hline
\end{tabular}

$$
B_{i j}=\left|\begin{array}{cccccc}
0.000017 & 0.000012 & 0.000007 & -0.000001 & -0.000005 & -0.000002 \\
0.000012 & 0.000014 & 0.000009 & 0.000001 & -0.000006 & -0.000001 \\
0.000007 & 0.000009 & 0.000031 & 0.000000 & -0.000010 & -0.000006 \\
-0.000001 & 0.000001 & 0.000000 & 0.000024 & -0.000006 & -0.000008 \\
-0.000005 & -0.000006 & -0.000010 & -0.000006 & 0.000129 & -0.000002 \\
-0.000002 & -0.000001 & -0.000006 & -0.000008 & -0.000002 & 0.000150
\end{array}\right|
$$

Table 2.Test results of proposed approach for 6- unit system.

\begin{tabular}{cccccccc}
\hline Load Demand (MW) & Pg1 & Pg2 & Pg3 & Pg4 & Pg5 & 50.0000 & 50.0000 \\
700 & 207.1793 & 50.0000 & 94.8030 & 50.0000 & 51.3818 & 75.8682 & 131.6575 \\
1000 & 285.9266 & 85.2588 & 155.6213 & 67.1309 & 107.3325 & 16000 \\
1200 & 355.4785 & 136.6740 & 209.5247 & 241.5669 & 140.7657 & 150.0000 & 193.4538 \\
1350 & 396.6995 & 167.1235 & 100.6272 & 120.0000 \\
1450 & 433.8446 & 196.6800 & 270.4180 & 150.0000 & 200.0000 & 120.0000 \\
\hline
\end{tabular}


Table 3. Performances analysis of the power and cost.

\begin{tabular}{ccccccc}
\hline \multirow{2}{*}{ Load Demand (MW) } & \multicolumn{2}{c}{ Existing } & \multicolumn{2}{c}{ EPSO } & \multicolumn{2}{c}{ Proposed } \\
\cline { 2 - 7 } & $\mathbf{P}_{\mathbf{L}} \mathbf{( M W )}$ & Fuel Cost (Rs) & $\mathbf{P}_{\mathbf{L}} \mathbf{( M W )}$ & Fuel Cost (Rs) & $\mathbf{P}_{\mathbf{L}}(\mathbf{M W})$ & Fuel Cost (Rs) \\
\hline 500 & 1.991 & 6105.02 & 1.98787 & 5498.17 & 1.9823 & 5221.2 \\
700 & 4.199 & 8287.55 & 4.25467 & 7756.21 & 4.0566 & 7068.8 \\
1000 & 8.123 & $11,930.40$ & 8.10602 & $11,312.9$ & 7.7980 & $10,147.0$ \\
1200 & 11.293 & $14,538.10$ & 11.1936 & $13,948.6$ & 11.1175 & $12,359.2$ \\
1350 & 14.086 & $16,575.50$ & 14.0436 & $15,982.6$ & 14.3965 & $14,102.3$ \\
1450 & 16.688 & $17,975.20$ & 16.6336 & $17,361.5$ & 16.7303 & $15,331.6$ \\
\hline
\end{tabular}

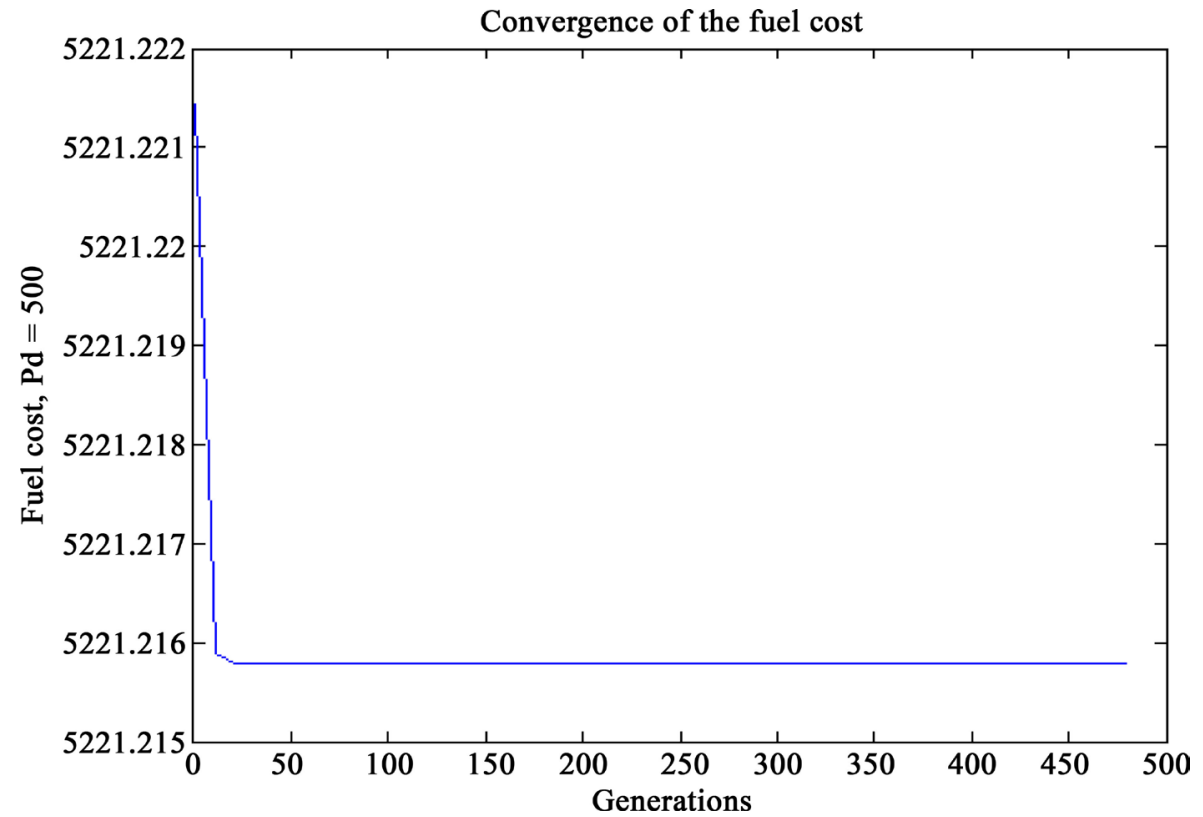

Figure 3. Simulation results of fuel cost for load demand 500.

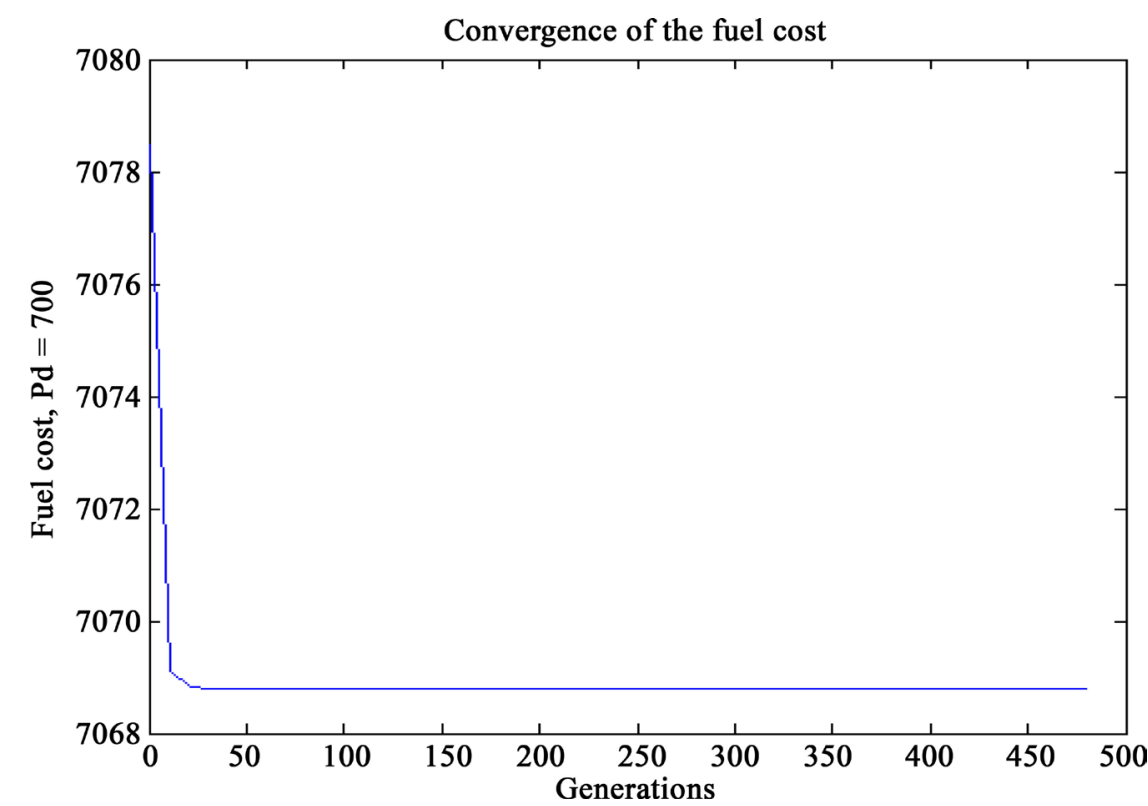

Figure 4. Simulation results of fuel cost for load demand 700. 


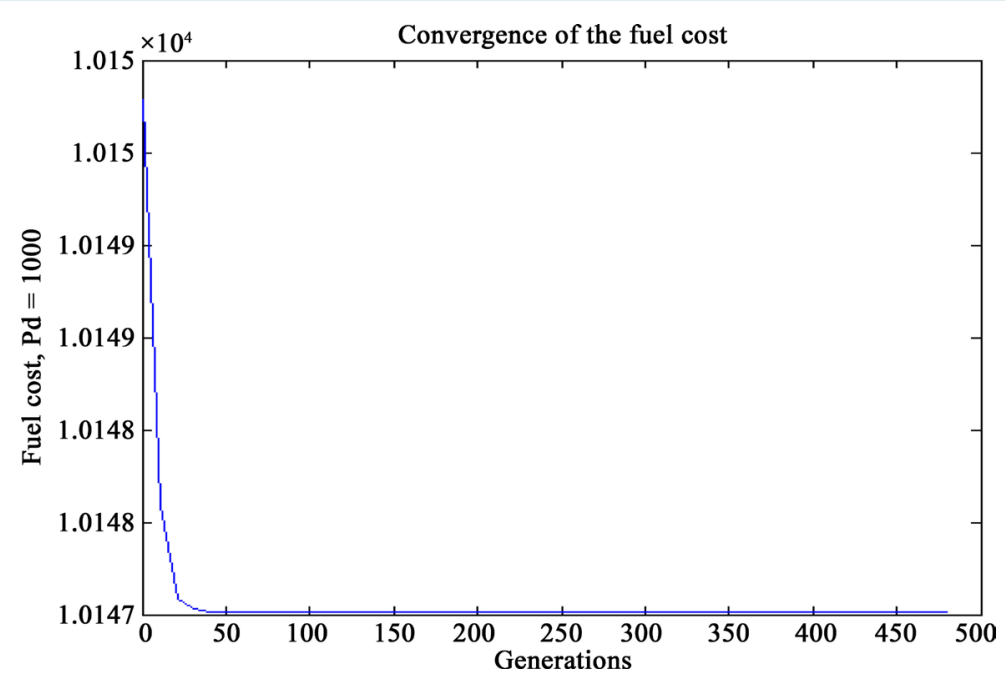

Figure 5. Simulation results of fuel cost for load demand 1000.

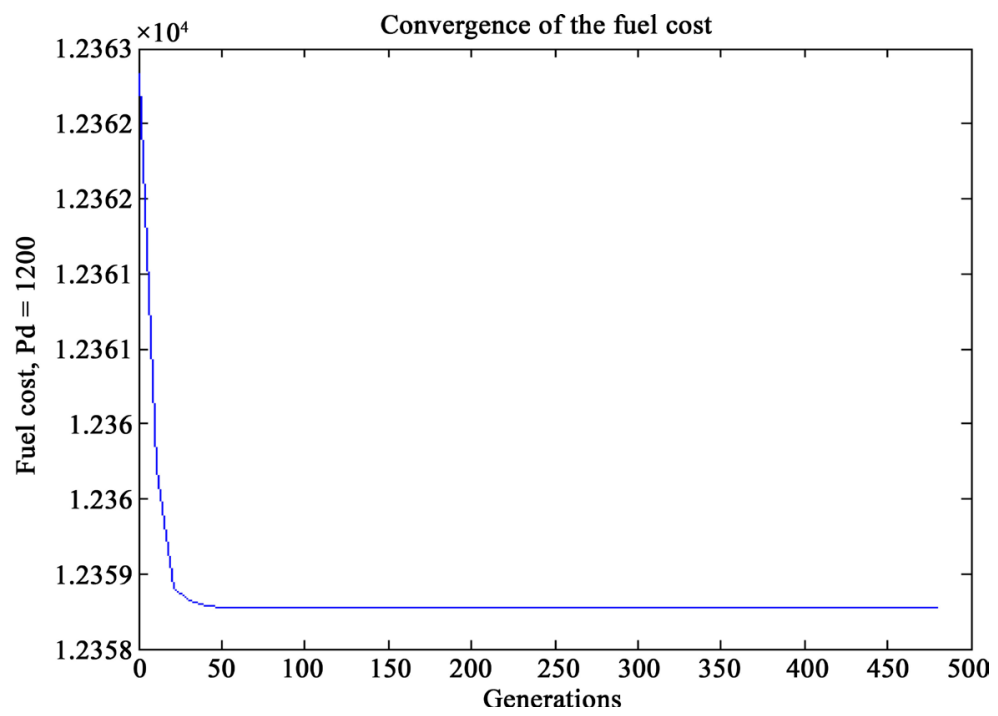

Figure 6. Simulation results of fuel cost for load demand 1200.

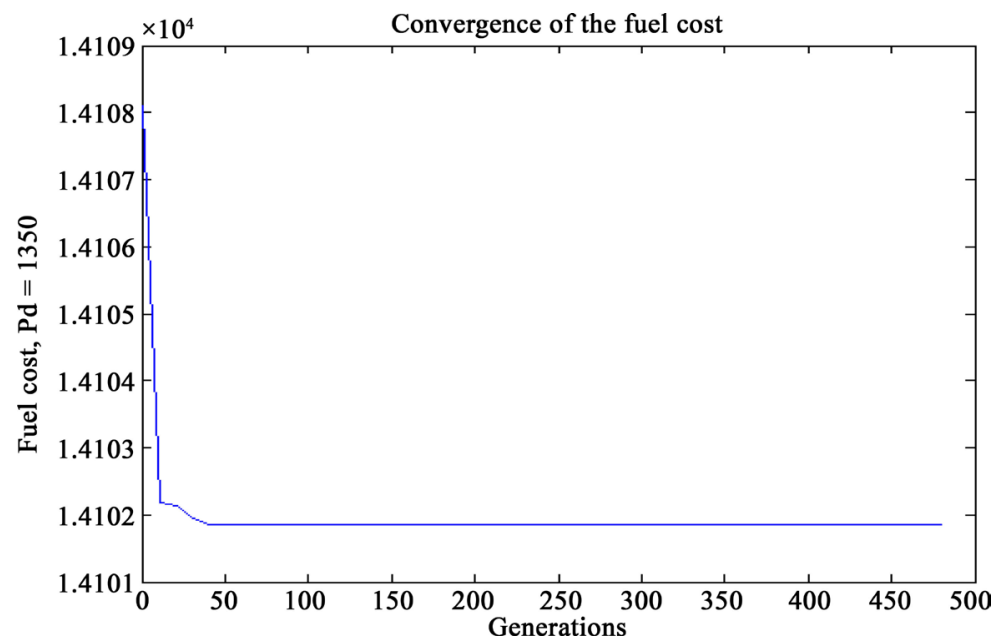

Figure 7. Simulation results of fuel cost for load demand 1350. 


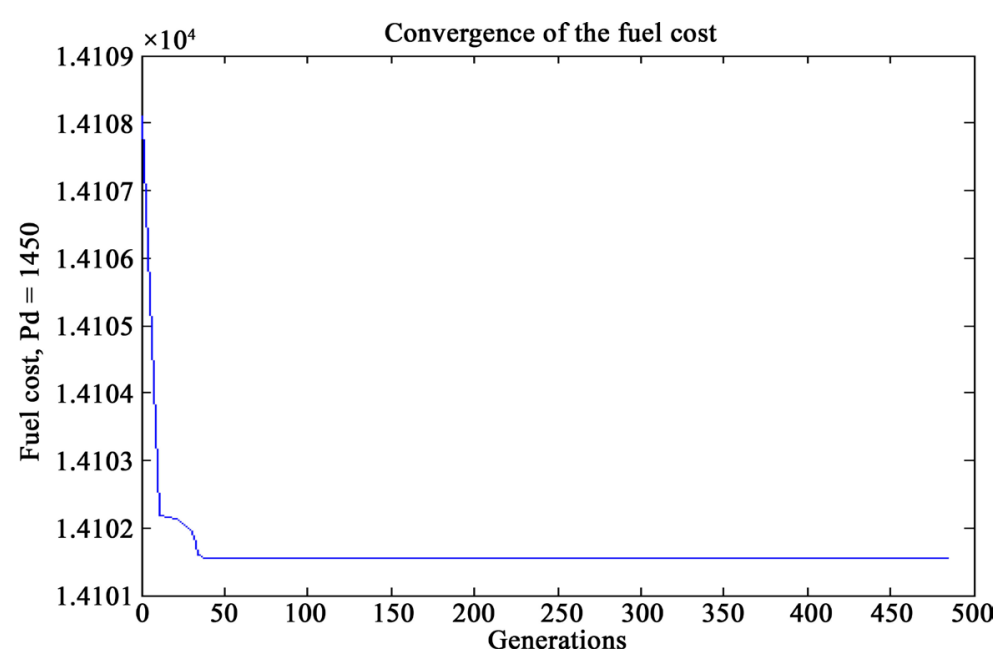

Figure 8. Simulation results of fuel cost for load demand 1450.

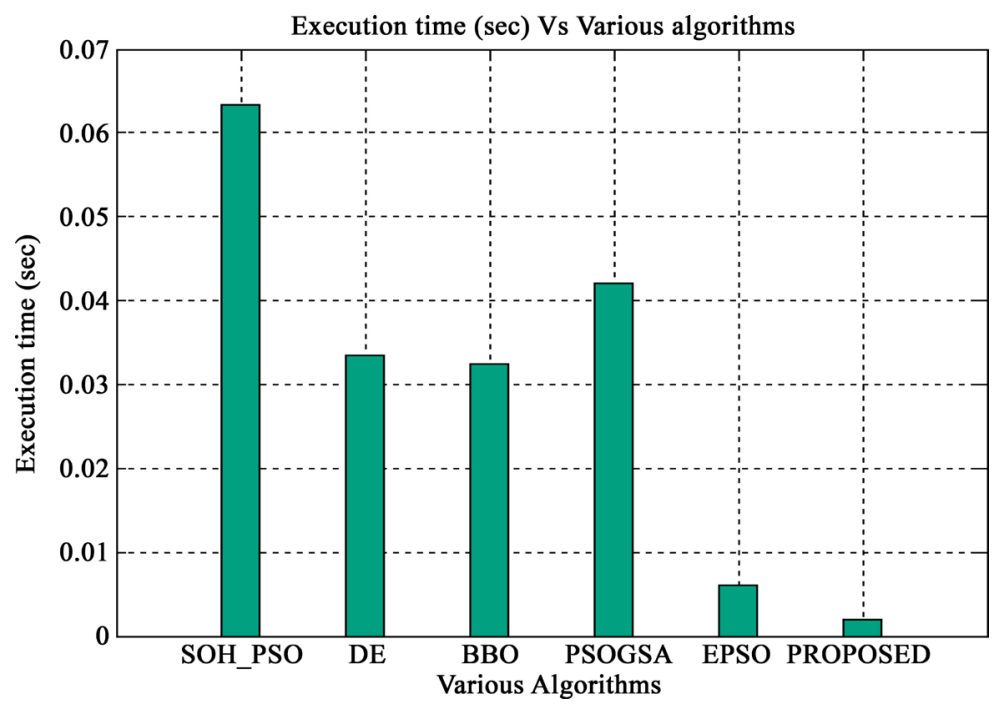

Figure 9. Simulation results of execution time vs various algorithm.

Table 4. Performances analysis of the time and minimum cost for various algorithm.

\begin{tabular}{ccc}
\hline Algorithm & Time/Iter (sec) & Min cost \\
\hline PSO & 14.89 & 15,450 \\
GA & 41.58 & 15,459 \\
NPSO-LRS & NA & 15,450 \\
ABF-NM & NA & $15,443.8164$ \\
DE & 0.0335 & $15,449.766$ \\
SOH-PSO & 0.0633 & $15,446.02$ \\
HHS & 0.14 & 15,449 \\
BBO & 0.0325 & $15,443.096$ \\
Hybrid SI-based HS & 0.9481 & $15,442.8423$ \\
PSOGSA & 0.0420 & $15,442.3930$ \\
EPSO & 0.0060 & $13,948.6$ \\
PROPOSED & 0.0055 & $13,948.6$ \\
\hline
\end{tabular}

Figure 9 shows the comparison of various algorithm execution times to obtain optimal solution. Table 4 illustrates the time requirement and the minimum cost for various algorithms. In that the proposed system obtained 
better performances than the existing algorithm. According to the procedure the results are evaluated to show the efficiency of the proposed approach.

\section{Conclusion}

In this paper, an Enhanced Hybrid Differential Evolution and Particle Swarm Optimization (EHDE-PSO) is proposed with Dynamic Sigmoid Weight in a parallel process. A hybrid form of the proposed approach combines the optimizing algorithm to have efficient Dynamic Economic Load Dispatch. It improves the information sharing and the accuracy to obtain the optimal solution from the issues, improves convergence and maintains diversity, finally it achieves less dropping probability. The simulation results show better efficiency of the proposed approach when compared with the existing. In the future, the dual evolution strategy with more performances should be improved.

\section{References}

[1] Kumar, V., Singh, J., Singh, Y. and Sood, S. (2015) Optimal Economic Load Dispatch Using Genetic Algorithms. International Journal of Electrical, Computer, Energetic, Electronic and Communication Engineering, 9, 440-447.

[2] Ramesh, B., Mohan, V.C.J. and Reddy, V.C.V. (2013) Application of Bat Algorithm for Combined Economic Load and Emission Dispatch. IJEETC, 2, 1-9.

[3] Karthikeyan, V., Senthilkumar, S. and Vijayalakshmi, V.J. (2013) A New Approach to the solution of Economic Dispatch Using Particle Swarm Optimization with Simulated Annealing. International Journal on Computational Sciences \& Applications (IJCSA), 3.

[4] Abou El Ela, A.A., Abido, M.A. and Spea, S.R. (2011) Differential Evolution Algorithm for Optimal Reactive Power Dispatch. Electric Power Systems Research, 81, 458-464. http://dx.doi.org/10.1016/j.epsr.2010.10.005

[5] Biswas, A., Dasgupta, S., Panigrahi, B.K., Pandi, V.R., Das, S., Abraham, A. and Badr, Y. (2009) Economic Load Dispatch Using a Chemotactic Differential Evolution Algorithm. 4th International Conference, HAIS 2009, Salamanca, 10-12 June 2009, 252-260. http://dx.doi.org/10.1016/j.apenergy.2009.10.007

[6] Alsumait, J.S., Sykulski, J.K. and Al-Othman, A.K. (2010) A hybrid GA-PS-SQP Method to Solve Power System Valve-Point Economic Dispatch Problems. Applied Energy, 87, 1773-1781.

[7] Alsumait, J.S. and Sykulski, J.K. (2009) Solving Economic Dispatch Problem Using Hybrid GA-PS-SQP Method. EUROCON 2009, EUROCON’09, St.-Petersburg, 18-23 May 2009, 333-338.

[8] Vlachogiannis, J.G. and Lee, K.Y. (2010) Closure to Discussion on "Economic Load Dispatch—A Comparative Study on Heuristic Optimization Techniques with an Improved Coordinated Aggregation-Based PSO. IEEE Transactions on Power Systems, 25, 591-592.

[9] Vlachogiannis, J.G. and Lee, K.Y. (2009) Economic Load Dispatch-A Comparative Study on Heuristic Optimization Techniques with an Improved Coordinated Aggregation-Based PSO. IEEE Transactions on Power Systems, 24, 991-1001.

[10] Hemamalini, S. and Simon, S.P. (2010) Dynamic Economic Dispatch with Valve-Point Effect Using Maclaurin Series Based Lagrangian Method. International Journal of Computer Applications, 1, 60-67.

[11] Premalatha, K. and Natarajan, A.M. (2009) Hybrid PSO and GA for Global Maximization. International Journal of Open Problems in Computer Science and Mathematics, 2, 597-608.

[12] Wang, S.-K., Chiou, J.-P. and Liu, C.W. (2007) Non-Smooth/Non-Convex Economic Dispatch by a Novel Hybrid Differential Evolution Algorithm. IET Generation, Transmission \& Distribution, 1, 793-803. http://dx.doi.org/10.1016/j.apenergy.2009.10.007

[13] Sayah, S. and Zehar, K. (2008) Using Evolutionary Computation to Solve the Economic Load Dispatch Problem. Leonardo Journal of Sciences, 12, 67-78.

[14] Wong, K.P. and Dong, Z.Y. (2005) Differential Evolution, an Alternative Approach to Evolutionary Algorithm. Proceedings of the 13th International Conference on Intelligent Systems Application to Power Systems, 6-10 November 2005, 73-83.

[15] Mahadevan, K., Kannan, P.S. and Kannan, S. (2005) Particle Swarm Optimization for Economic Dispatch of Generating Units with Valve-Point Loading. Journal of Energy \& Environment, 4, 49-61.

[16] Abido, M.A. (2003) A novel Multiobjective Evolutionary Algorithm for Environmental/Economic Power Dispatch. Electric Power Systems Research, 65, 71-81. http://dx.doi.org/10.1016/S0378-7796(02)00221-3 


\section{Submit or recommend next manuscript to SCIRP and we will provide best service for you:}

Accepting pre-submission inquiries through Email, Facebook, LinkedIn, Twitter, etc.

A wide selection of journals (inclusive of 9 subjects, more than 200 journals)

Providing 24-hour high-quality service

User-friendly online submission system

Fair and swift peer-review system

Efficient typesetting and proofreading procedure

Display of the result of downloads and visits, as well as the number of cited articles

Maximum dissemination of your research work

Submit your manuscript at: http://papersubmission.scirp.org/ 